\title{
Rhizospheric microbiomes of Sorghum bicolor grown on soils with anthropogenic polyelement anomalies
}

\author{
Anna Yu.Muratova ${ }^{1, *}$, Svetlana $V$. Gorelova ${ }^{2}$, Irina Yu. Sungurtseva ${ }^{1}$, and Nadezhda A. \\ Zelenova $^{3}$ \\ ${ }^{1}$ Institute of Biochemistry and Physiology of Plants and Microorganisms, RAS, Laboratory of \\ Environmental Biotechnology, 410049 Saratov, Russia \\ ${ }^{2}$ Tula State University, Natural Science Institute, Department of Biology, 300012 Tula, Russia \\ ${ }^{3}$ Saratov State University, Department of Biology, 410012 Saratov, Russia
}

\begin{abstract}
Comparative study was made of the rhizospheree microbiomes of two cultivars of sorghum (Sorghum bicolor cvs. Sucro 506 and Biomass 133) grown on soils with anthropogenic polyelement anomalies and on a background (control) soil. The study used traditional culture-based and culture-independent metagenomic approaches. In soils contaminated with heavy metals, we found decreased numbers of culturable bacteria and quantitative changes in the populations of actinomycetes and micromycetes. The relative abundance of the families whose members were able to resist heavy metals was found to increase in the rhizospheric communities. The taxonomic profile of the microbial communities at the phylum level did not differ significantly between cultivars. The Shannon diversity and the abundance of actinomycete families in the rhizosphere of cv. Biomass 133 were greater than those for cv. Sucro 506. Significant differences were found between cultivars for the number of rhizospheric microorganisms resistant to heavy metals.
\end{abstract}

\section{Introduction}

Interactions between plants and their associated microorganisms have a global impact on many biogeochemical processes in nature, including the mineralization of organic substances and the element cycles. The rhizosphere is a unique niche saturated with the physical, chemical, and biological interactions between macro- and micropartners and between these and the environment. In the past few decades, research in rhizosphere biology has not waned; instead, it has even intensified, because it allows one to tackle urgent problems such as those of increasing crop yields and improving the use of plants and microorganisms to restore disturbed and polluted environments (phytoremediation). One current trend in rhizosphere biology is to elucidate microbiome formation in the plant root zone. For successful phytoremediation, it is fundamentally important to develop plant-microbial associations that would intensely decompose organic pollutants and transform, sequester, and/or accumulate

${ }^{*}$ Corresponding author: muratova a@ibppm.ru 
inorganic ones. In this respect, it is important to study the structure of the rhizosphere microbiome, as well as to choose the appropriate plant type and variety as the basis for a plant-microbial association.

Besides being used in traditional agriculture, Sorghum bicolor (L.) Moench. is used widely in the remediation of heavy-metal-contaminated soils [1-3]. The microbial communities of the sorghum rhizosphere have been examined in earlier studies [4-6], which have shown that their structure is affected by abiotic (soil, drought) and biotic (bacterial inoculation) factors. The influence of the plant genotype and development stage on the composition of the sorghum rhizosphere microbiome has been reported [5-7]. The rhizosphere communities of sorghum contain a sufficient number of taxa to enhance plant growth [4-6, 8]. However, little work has been done to examine the influence of heavy metals on microbiome formation in the sorghum rhizosphere and on the rhizospheric microorganisms resistant to heavy metals and involved in their conversion. Yet, a change in the relative abundance of individual taxa in soil microbial communities may indicate soil contamination and characterize soil restoration potential [9-11].

Here we made a comparative study of the rhizosphere microbiomes of two S. bicolor cultivars grown on soils contaminated with heavy metals.

\section{Materials and methods}

\subsection{Objects of study}

We used samples of the rhizosphere soil of S. bicolor cvs. Sucro 506 and Biomass 133. The plants were grown for six months under laboratory conditions in 2-liter plastic containers filled with three types of industrially contaminated soils and with a background (control) soil. The experimental soils, sampled in the city of Tula, were urban soils based on gray forest soils. They had neutral or close to neutral $\mathrm{pH}$ values and a high Fe content. The soils sampled in industrial (sanitary protection zones of the PJSC Kosogorsky Metallurgical Plant [KMP] and the PJSC Tulachermet [TChM]) and urban (Lenin Avenue [LP]) areas exhibited polyelement anomalies. The KMP soil had a high content of Fe $(78,100 \mathrm{mg} / \mathrm{kg})$ and the exceeded maximum permissible concentration (MPC) for Mn (3.8-4.4-fold) and Zn (by $41 \%)$. The TChM soil had a very high Fe content $(120,600 \mathrm{mg} / \mathrm{kg})$ and the exceeded MPCs for V-Mn (by $40-50 \%$ for $\mathrm{V}$ and $10 \%$ for $\mathrm{Mn}$ ), $\mathrm{Ni}$ (by 110-175\%), Cu (by 127\%), Zn (by 29-192\%), As (by 115-220\%), and petroleum products (4-fold). The LP soil had a high content of Fe $(37,400 \mathrm{mg} / \mathrm{kg}$ ) and the exceeded MPCs for Mn (by 6\%) and $\mathrm{Cu}$ (by 186\%). The control soil was collected from the grounds of the Yasnaya Polyana Museum-Estate of Leo Tolstoy, located $14 \mathrm{~km}$ southwest of Tula. Although the Fe content of the control soil was rather high, as compared to the world values, but minimal $(15,600 \mathrm{mg} / \mathrm{kg})$, as compared to the urban and industrial soil values, the control soil did not have polyelement anomalies.

\subsection{Microbiological analyses of rhizosphere communities}

The total numbers of culturable heterotrophic bacteria, actinomycetes, and micromycetes after plant growth were estimated by a microbiological analysis of the rhizosphere soil, which was conducted by the plating method. Bacteria were counted on beef-extract agar; actinomycetes, on starch-ammonia agar; and micromycetes, on Martin's agar. Heavy-metalresistant microorganisms were counted on the LB agar medium, which contained the watersoluble salts $\mathrm{ZnSO}_{4}, \mathrm{CuSO}_{4}$, and $\mathrm{Pb}\left(\mathrm{NO}_{3}\right)_{2}$ (concentrations of $\mathrm{Zn}^{2+}, \mathrm{Cu}^{2+}$, and $\mathrm{Pb}^{2+}$ ions, 0.5 $\mathrm{mmol} / \mathrm{L})$. The inoculated plates were incubated at $28-30^{\circ} \mathrm{C}$ for $5-10$ days, after which microbial colonies and CFU were counted and the morphological diversity of the 
microorganisms was measured. Colonies of different morphological types grown with metal ions were used as pure cultures. Data were processed with Microsoft Excel 2007 (Microsoft, USA).

The taxonomic structure of the rhizosphere communities associated with sorghum was studied by16S rRNA gene-based metagenomic analysis. Total bacterial genomic DNA was extracted from soil samples by using a Fast DNA SPIN kit for soil (Qbiogene, Heidelberg, Germany) according to the manufacturer's instructions. Sequencing was done on the Illumina MiSeq platform. Data were analyzed on the QIIME platform, v. 1.9.1.

\section{Results and discussion}

\subsection{Culture-based estimation of rhizosphere microbial communities}

The results for the main groups of culturable heterotrophic microorganisms (bacteria, actinomycetes, and micromycetes) in the rhizosphere of the two sorghum cultivars are shown in Fig.1. When cv. Sucro 506 was grown on contaminated soils, the number of bacteria decreased greatly on all soils (by 75-91\%), that of actinomycetes decreased to a lesser extent on all soils (by $36-86 \%$ ), and that of micromycetes increased by $55 \%$ on the TChM soil, decreased by $87 \%$ on the LP soil, and changed non significantly on the KMP soil. When cv. Biomass 133 was grown on contaminated soils, the number of heterotrophic bacteria decreased by $73-86 \%$ on all soils, that of actinomycetes decreased by $55 \%$ on the TChM soil (or changed non significantly on the KMP and LP soils), and that of micromycetes decreased severely on the TChM soil (by 90\%) and less severely on the KMP and LP soils.

A

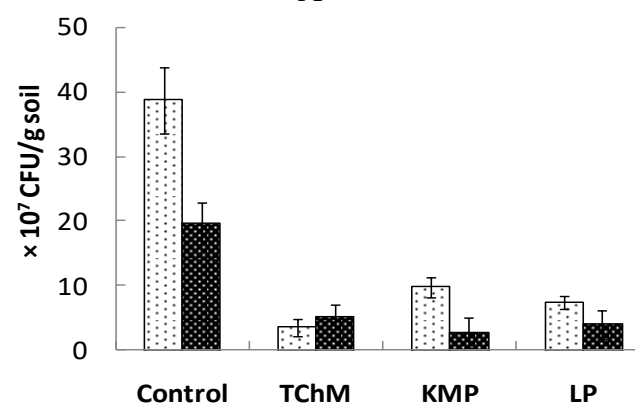

$\mathrm{C}$

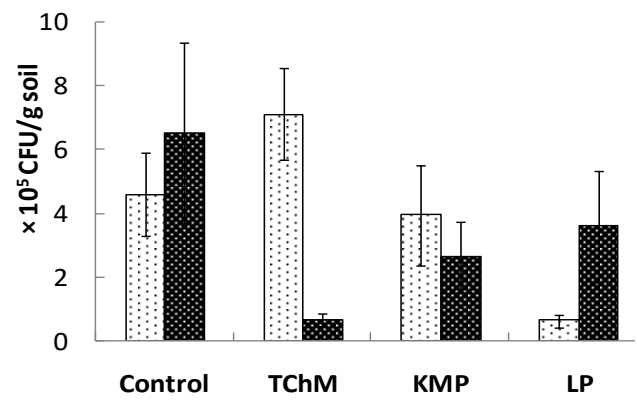

B

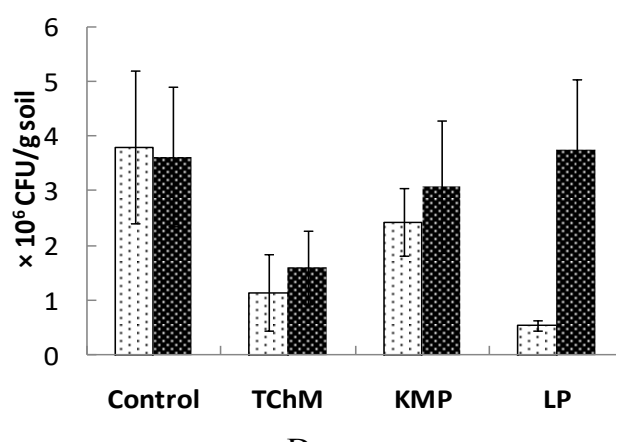

$\mathrm{D}$

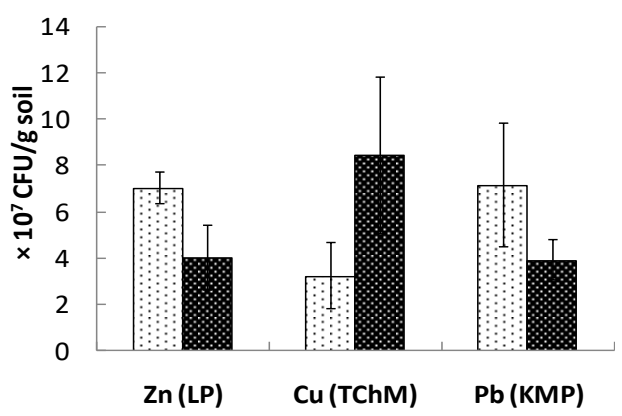

Fig. 1. Total numbers of culturable heterotrophic microorganisms in the rhizosphere of cvs. Sucro 506 and Biomass 133. A, bacteria; B, actinomycetes; C, micromycetes; D, metal-resistant microorganisms; $\because$,cv. Sucro 506; $\mathbf{2}$,cv. Biomass 133 
The observed decreases in the numbers of rhizospheric microorganisms are obviously due to the high content of heavy metals in the soils. Although metals function as trace elements in the vital activity of soil microorganisms, their high concentrations are toxic to the microbiota. The toxicity of metals to soil bacteria is associated with disruption of redox reactions, damage to the enzymes, and changes in the conformational structure of nucleic acids and proteins. All these changes give rise to complexes that make protein molecules inactive [12].

In contrast to actinomycetes and fungi, bacteria are more sensitive to soil pollution by metals [12-14]. This was observed in our study. However, the effect of metal pollution on the rhizosphere numbers of actinomycetes and micromycetes differed between cultivars and was most noticeable on the LP and TChM soils (Fig. $1 \mathrm{~B}, \mathrm{C}$ ). The intercultivar differences in the formation of rhizosphere communities of actinomycetes and micromycetes in soils with anthropogenic polyelement anomalies may be due to the main characteristics of these soils [6], to the extent of contamination, and probably to the differences in the composition of the root exudates, which are essential for the formation of a specific rhizosphere microbiome [57].

A comparison of the numbers of resistant rhizospheric microorganisms revealed significant intercultivar differences (Fig. $1 \mathrm{D}$ ). The numbers of microorganisms resistant to $\mathrm{Zn}$ and $\mathrm{Pb}$ in the rhizosphere of Biomass 133 were 75 and $81 \%$ smaller than those in the rhizosphere of Sucro 506, respectively. The number of microorganisms resistant to $\mathrm{Cu}$ was $62 \%$ larger than that in the rhizosphere of Sucro 506. As a result, we assembled a collection of 32 microbial isolates resistant to heavy metals.

\subsection{Metagenomic analysis of rhizosphere microbial communities}

Sequencing of the 16s rRNA gene from the sorghum rhizosphere samples resulted in 924,685 reads. After data denoising and chimera screening, 273,890 sequences were used for further identification. The sequences with a $>97 \%$ similarity were combined into operational taxonomic units (OTUs). A total of 208,127 OTUs were identified across all the samples. The OTUs were assigned to $45,158,300,495$, and 852 taxa at the phylum, class, order, family, and genus levels, respectively.

In order to characterize the biodiversity and comparatively analyze the microbial communities, we calculated the parameters of $a-$ and $\beta$-diversity, compared the taxonomic compositions of all samples, and identified the taxa that exhibited statistically significant decreases or increases in number in the plant rhizosphere. The a-diversity was measured by using the species richness indices (the OTU value in the sample) and the Shannon index.

Calculation of the sampling effort indicated that on average, 73 and $68 \%$ of the true diversity of the rhizosphere communities of cvs. Sucro 506 and Biomass 133, respectively, were covered. The average number of OTUs in the rhizosphere of cv. Biomass $133(7,213$ on average) was greater than in the rhizosphere of cv. Sucro 506 (6,314 on average). The obtained data indicated that the species richness index of Biomass 133 was higher than that of Sucro 506.

In order to measure a-diversity, we calculated the Shannon index (11.1674 and 11.4928) and the Simpson index (0.9983 and 0.9989) for cvs. Sucro 506 and Biomass 133, respectively. The values of these indices turned out close between cultivars and indicated a high taxonomic diversity of the rhizosphere microbial communities. As reported earlier, the diversity indices of the rhizosphere microbial communities do not differ from those of the microbial communities of bulk soils, both in the analysis of different types of soils and varieties [15-16] and in the analysis of the rhizospheres of plants of different ages [15].

A total of 45 taxa at the phylum level were identified across samples, of which 12 were represented by at least $1 \%$ of the total reads. The phylum level distribution across samples 
for these taxa is presented in Fig 2. It can be seen that the taxonomic profile of the rhizosphere communities at the phylum level did not differ significantly between cultivars and between plants grown on contaminated and uncontaminated soil. However, the taxonomic profile of the sorghum rhizosphere differed significantly from that of the rhizosphere microbiome of another plant, Amaranthus, grown under the same conditions (data not shown). This indicates differences in the taxonomic structure of the rhizosphere communities of plants of different classes and the storage of species-specific taxonomic profiles of the rhizosphere microbiomes in soils differing in the extent of contamination.

In the rhizosphere communities of the cultivars, most OTUs were assigned to the Actinobacteria (29 and 30\% for Sucro 506 and Biomass 133, respectively) and Proteobacteria (27 and 26\% for Sucro506 and Biomass 133, respectively). A significantly smaller percentage of OTUs was assigned to other phyla, in descending order for cvs. Sucro506 and Biomass 133, respectively: Chloroflexi (9 and 10\%), Acidobacteria (9\% each), Planctomycetes (5 and 6\%), and Bacterioides (4.7 and $3.5 \%$ ). The rhizosphere microbiome of cv. Sucro 506 grown on the KMZ soil contained more than $6 \%$ of the Firmicutes phylum, members of which are often found in soils contaminated with heavy metals [17].

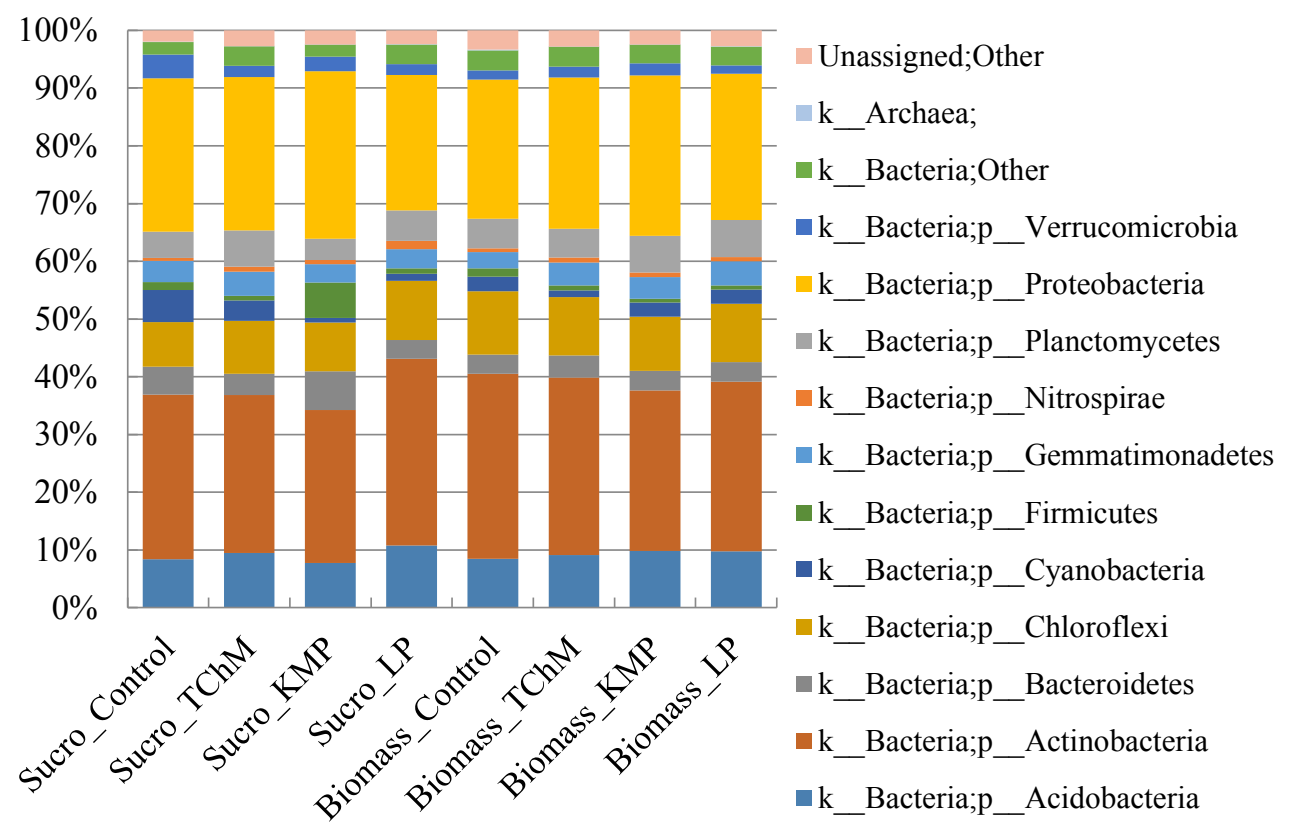

Fig.2. Phylum-level relative abundances of the microbial communities in the rhizosphere of cvs.

Sucro 506 and Biomass 133 grown on the background (control) soil and on soils with anthropogenic polyelement anomalies

Of the Actinobacteria phylum, 60 taxa were identifiedat the family level, of which 11 occupied the principal position, making up $67 \%$ to $80 \%$ of all detected actinobacterial families in the sorghum rhizosphere. Most of the OTUs identified in the rhizosphere actinomycete population belonged to the Gaiellaceae family, reaching $2.6-5.5 \%$ for cv. Sucro 506 and $4.6-6.4 \%$ for cv. Biomass 133. Previously, Sun et al. [18] suggested a potential ecological role for Gaiellaceae bacteria in metal-contaminated soils, revealing their increased numbers in sequencing libraries and a close significant correlation with various metals or metalloids. When sorghum plants were grown on metal-contaminated soils, the percentage of members of another family of Actinobacteria (C111) increased in the rhizosphere of both cultivars. In the rhizosphere of cv. Biomass 133, the number of OTUs assigned to C111 was 
larger than that in the rhizosphere of cv. Sucro 506 (1.4 and 2.5 on the control soil and 2.9 and 3.1 on the contaminated soil, respectively). The actinomycete populations of sorghum also included families belonging to the Acidimicrobiales, Solirubrobacterales, and 0319$7 \mathrm{~L} 14$ orders. In general, the percentage of the actinomycete families identified in the rhizosphere of cv. Biomass 133 was higher than that in the rhizosphere of cv. Sucro 506. These data are consistent with the greater number of culturable actinomycetes in the rhizosphere of cv. Biomass 133, as found by culture-based microbiological analysis (Fig. 1).

Of the Proteobacteria phylum, 113 taxa were identified at the family level, of which 12 made the most significant contribution to the structure of the microbiomes of the two cultivars. The Alphaproteobacteria was the major class, accounting for $46 \%$ of all OTUs assigned to the Proteobacteria. Among the Alphaproteobacteria, the Rhizobiales order, with the dominant Hyphomicrobiaceae (2.4-4.4\%) and Bradyrhizobiaceae (0.8-1.7\%) families, had the greatest taxonomic diversity. The percentage of OTUs assigned to the Hyphomicrobiaceae was the largest. In the rhizosphere of cv. Sucro 506, it was maximal on uncontaminated soil; in the rhizosphere of cv. Biomass133, on the contrary, the largest percentage of OTUs was observed on the KMP and LP soils. Within the Alphaproteobacteria class, the Rhodospirillaceae and Sphingomonadaceae families made a significant contribution to the formation of the rhizosphere microbiomes. The Betaproteobacteria class accounted for $32 \%$ of all OTUs assigned to the Proteobacteria, with members of the Comamonadaceae family being predominant (1.0-2.3\%). The relative abundance of OTUs assigned to this family was maximal in the rhizosphere of plants grown on uncontaminated soil, but it decreased on contaminated soils. The contribution of the Deltaproteobacteria and Gammaproteobacteria classes to the general taxonomic structure of the sorghum microbiomes was noticeably modest -5 and $3 \%$ of all OTUs assigned to the Proteobacteria, respectively.

The growth of sorghum on contaminated soils was accompanied by a decrease in the percentage of the Cytophagaceae family (order Cytophagales, class Cytophaga, phylum Bacteriodes), particularly on the LP soil (by 78 and 30\% in the rhizosphere of cvs. Sucro 506 and Biomass 133, respectively).

In summary, this study has found qualitative and quantitative changes in the rhizospheric microbial communities formed under the influence of the S. bicolor genotype and humancaused pollution of soil. The rhizosphere microflora of cv. Biomass 133 was more resistant to $\mathrm{Cu}$, and that of $\mathrm{cv}$. Sucro 506 was more resistant to $\mathrm{Zn}$ and $\mathrm{Pb}$. These findings should be taken into account in the phytoremediation of soils with elemental anomalies. As a result of the study, a collection of 32 heavy-metal-resistant isolates was assembled. A more detailed study of how the isolated strains resist metals and promote plant growth would make it possible to choose a promising inoculant to improve the growth of sorghum on contaminated soils and increase the efficiency of phytoremediation.

This study was supported by the Russian Foundation for Basic Research (project no. 19-29-05257).

\section{References}

1. L. Marchiol, G. Fellet, D. Perosa, G. Zerbi, Plant Physiol. Biochem. 45, 379 (2007). DOI: 10.1016/j.plaphy.2007.03.018

2. P. Zhuang, W. Shu, Z. Li, B. Liao, J. Li, J. Shao, J. Environ. Sci. 21, 1432 (2009). DOI: 10.1016/s1001-0742(08)62436-5

3. P. Soudek, Š. Petrová, R. Vaňková, J. Song, T. Vaněk, Chemosphere 104, 15 (2014). DOI: $10.1016 /$ j.chemosphere.2013.09.079 
4. F. Tshabuse, Identification of rhizospheric microorganisms associated with sorghum (University of the Western Cape, Cape Town, 2012)

5. T. R. Schlemper, J. A. van Veen, E. E. Kuramae, Microb. Ecol. 76, 205 (2018). DOI: 10.1007/s00248-017-1108-6

6. T. R. Schlemper, M. F. A. Leite, A. R. Lucheta, M. Shimels, H. J. Bouwmeester, J. A. van Veen, E. E. Kuramae, FEMS Microbiol. Ecol. 93 (2017). DOI: 10.1093/femsec/fix096

7. S. Deng, D. Caddell, J. Yang, L. Dahlen, L. Washington, D. Coleman-Derr, bioRxiv (2020). DOI: 10.1101/2020.02.21.960377

8. T. Oberholster, S. Vikram, D. Cowan, A. Valverde, Sci. Total Environ, 624, 530 (2018). DOI: $10.1016 /$ j.scitotenv.2017.12.170

9. F. Gremion, A. Chatzinotas, K. Kaufmann, W. V. Sigler, H. Harms, FEMS Microbiol. Ecol. 48, 273 (2004). DOI: 10.1016/j.femsec.2004.02.004

10. L. Epelde, I. Mijangos, J. M. Becerril, C. Garbisu, Soil Biol. Biochem. 41, 1788 (2009). DOI: 10.1016/j.soilbio.2008.04.001

11. S. M. Hermans, H. L. Buckley, B. S. Case, F. Curran-Cournane, M. Taylor, G. Lear, Appl. Environ. Microbiol. 83 (2017). DOI: 10.1128/AEM.02826-16

12. A. Lenart-Boro, P. Boro, Environmental risk assessment of soil contamination (InTech, London, UK, 2014)

13. V. N. Grishko, O. V. Syshchikova, Eurasian Soil Sc. 42, 217 (2009). DOI: $10.1134 / \mathrm{S} 1064229309020136$

14. I. G. Shirokikh, E. S. Solov'eva, T. Y. Ashikhmina, Eurasian Soil Sc. 47, 89 (2014). DOI: $10.1134 / \mathrm{S} 1064229313100062$

15. J. M. Chaparro, D. V. Badri, J. M. Vivanco, The ISME Journal 8, 790 (2014). DOI: 10.1038/ismej.2013.196

16. J. Edwards, C. Johnson, C. Santos-Medellín, E. Lurie, N. K. Podishetty, S. Bhatnagar, J. A. Eisen, V. Sundaresan, Proc. Nat. Acad. Sci. USA 112, E911 (2015). DOI: 10.1073/pnas.1414592112

17. C. Pires, A. R. Franco, S. I. A. Pereira, I. Henriques, A. Correia, N. Magan, P. M. L. Castro, Geomicrobiol. J. 34, 760 (2017). DOI: 10.1080/01490451.2016.1261968

18. W. Sun, E. Xiao, V. Krumins, M. M. Häggblom, Y. Dong, Z. Pu, B. Li, Q. Wang, T. Xiao, F. Li, Appl. Environ. Microbiol. 84, e00701 (2018). DOI: 10.1128/AEM.0070118 